\title{
TOWARDS AN EMPIRICAL MODEL TO ESTIMATE THE SPATIAL VARIABILITY OF GRAPEVINE PHENOLOGY AT THE WITHIN FIELD SCALE
}

\author{
N. Verdugo-Vásquez ${ }^{1}$, C. Acevedo-Opazo ${ }^{2 *}$, H. Valdés-Gómez ${ }^{3}$, B. Ingram ${ }^{4}$, I. García de Cortázar-

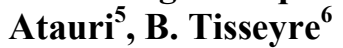 \\ ${ }^{1}$ Instituto de Investigaciones Agropecuarias INIA, Centro de Investigación Intihuasi, Colina San Joaquín s/n, P.O. \\ Box 36-B, La Serena, Chile. \\ ${ }^{2}$ Universidad de Talca, Facultad de Ciencias Agrarias, CITRA, 2 Norte, 685 Talca, Chile. \\ ${ }^{3}$ Pontificia Universidad Católica de Chile, Departamento de Fruticultura y Enología, Vicuña Mackenna 4860, \\ Santiago, Chile. \\ ${ }^{4}$ Cranfield Soil and Agrifood Institute, Cranfield University, College Road, Cranfield, Bedfordshire, MK43 0AL, \\ UK. \\ ${ }^{5}$ INRA, US 1116 AGROCLIM, 84914 Avignon, France. \\ ${ }^{6}$ ITAP, Montpellier SupAgro, Irstea, Univ. Montpellier Bât. 21, 2 P1. Pierre Viala, Montpellier 34060, France. \\ *Corresponding author: e-mail: cacevedo@utalca.cl
}

\begin{abstract}
The aim of this study is to propose an empirical spatial model to estimate the spatial variability of grapevine phenology at the within-field scale. This spatial model allows the characterization of the spatial variability of the fields through a single measurement performed in the field (reference site) and a combination of site-specific coefficients calculated through historical data. This approach was compared to classical approaches requiring extensive sampling and phenology models based on climatic data, which do not consider the spatial variability of the field. The study was conducted on two fields, one of cv Cabernet Sauvignon (CS, 1.56 ha) and the other one of cv Chardonnay (CH, 1.66 ha) located in the Maule Valley, Chile. Measurements of the date of occurrence of grapevine phenology (budburst, flowering and veraison) were observed at the within field level following a regular sampling grid (18 sites for cv CS and 19 sites for $\mathrm{cv} \mathrm{CH}$ ) during 4 seasons for $\mathrm{cv} \mathrm{CS}$ and 2 seasons for $\mathrm{cv} \mathrm{CH}$. The spatial model was calibrated using data collected during the first 3 seasons of $\mathrm{cv} \mathrm{CS}$ and the 2 seasons of $\mathrm{cv} \mathrm{CH}$, while the last season of cv CS was used for the validation process. Regarding the quality of estimation, the best results were obtained with the spatial model in almost all cases, with a Root Mean Square Errors (RMSE) lower than 3 days. However, if the variability of phenology is low, the traditional method of sampling could lead to better results. This study is the first step towards a modeling of the spatial variability of grapevine phenology at the within-field scale. To be fully operational in commercial vineyards, the calibration process needs simplification, for example, using low cost, inexpensive ancillary information to zone vineyards according to grapevine phenology. This study opens up the opportunity to combine classical models of phenology based on climatic data with spatial models, with the aim of predicting the grapevine phenology both in time and space.
\end{abstract}

Keywords: Spatial model, Climatic models, Extensive sampling, Sensitivity analysis. 


\section{Introduction}

In recent years, several authors have shown that there is significant spatial variability in vineyards for many variables at the within-field scale (Arnó et al. 2009; Tisseyre et al. 2008). These variables are related to environmental parameters of the vineyards such as soil (Taylor et al. 2009), microclimate conditions (Matese et al. 2014) and plant characteristics such as water status (Acevedo-Opazo et al. 2010), vegetative growth (King et al. 2014), vine yield (Arnó et al. 2011; Taylor et al. 2005), grape chemical composition (Baluja et al. 2013; Bramley et al. 2011), plant diseases (Bramley et al. 2011) and precocity of the phenology (Verdugo-Vásquez et al. 2016). This last study showed that under certain conditions the within-field spatial variability of grapevine phenology may be as important as at the regional scale and may justify site-specific management practices. Knowledge and monitoring of the grapevine phenology throughout the season are important requirements for planning many productive activities (Mullins et al. 1992). For example, practices such as irrigation, fertilization, phytosanitary application and harvest are scheduled based on the monitoring and the evolution of the grapevine phenology. For proper planning of the practices mentioned above, vine-growers need to monitor and know in advance the date of occurrence of the key phenological stages. Therefore, due to this observed spatial variability, new challenges arise in the monitoring and modeling of grapevine phenology at the within-field scale. Traditionally, the method used by vine-growers in their vineyards consists in making two or three phenological observations per field. These observations are assumed to be representative of the whole vineyard and sometimes of other neighboring fields of the same cultivar (Verdugo-Vásquez et al. 2016). Thus, traditional methods would not be appropriate to represent the spatial variability of the fields and therefore could result in inadequate intervention decisions.

To model grapevine phenology, climate variables such as air temperature are used to predict phenological stages (Chuine et al. 2013). The relationship between phenology and air temperature has led to classical climatic models of phenology to be proposed, mainly developed in the Northern Hemisphere (Caffarra and Eccel 2010; García de Cortázar-Atauri et al. 2009; Molitor et al. 2013; Parker et al. 2011) and in some cases in the Southern Hemisphere (Jorquera-Fontena and Orrego-Verdugo 2010; Ortega-Farías et al. 2002; Verdugo-Vásquez et al. 2017). These climatic models predict the date of occurrence of the key phenological stages (for example budburst, flowering and/or veraison) using air temperature as the only variable. Thus, by means of these climatic models it is possible to estimate the date of occurrence of the phenological stages. Temperature data used for these models usually come from meteorological stations or service providers. Whatever their origin, meteorological data are usually available at a medium or low spatial resolution, therefore depending on the vineyard location (and environmental attributes), air temperature used to calculate the results from the models may differ from the real air temperature 
observed in the field, as described by Matese et al. (2014). In addition, data resolution is too low to be considered for the within-field scale level; as a result, meteorological data are generally applied at the vineyard or at the field scale. Therefore, climatic models provide an average value of the date of occurrence of the phenological stage, which is extrapolated to the whole vineyard without considering the spatial variability. Under conditions of high spatial variability, the extrapolation of the average value obtained through the climatic models may not be representative of the whole fields. An approximation of the climatic model that considers the spatial variability could be microclimate monitoring of the field using a wireless temperature sensors network. However, this monitoring approach presents several drawbacks: it is difficult to define the number and the location of sensors to be installed in the field and the maintenance cost of the system may limit its practical application in the vineyards (Kunz and Tatham 2012; Primicerio et al. 2013).

An alternative approach to overcome the above problem is the use of an empirical spatial model. The empirical spatial model can be used to estimate a variable over the whole field while taking into account the spatial variability. This kind of approach has been applied successfully to estimate vine water status in non-irrigated (Acevedo-Opazo et al. 2010) and irrigated conditions (Acevedo-Opazo et al. 2013). It relies on the calibration of an observation collected in the field (the reference site) with a combination of sitespecific coefficients estimated using historical data. Such an approach motivates the interest in the temporal evolution of a parameter obtained from measurements collected at the within field level whereas spatial variability is modeled by site-specific coefficients for the extrapolation of measurements at the reference site. This approach might be interesting for the estimation of the precocity of the grapevine phenology (delay or advance of the date of occurrence of phenological stages) with respect to the phenology observed at a reference site. A recent study (Verdugo-Vásquez et al. 2016) showed that there is a high temporal stability of the within-field variability of the vine phenology under semi-arid conditions. This knowledge opens up the possibility of using ancillary data of phenology (historical data) measured at the within-field scale with the aim of developing empirical methods for the calibration of spatial models. The advantage of this approach is that spatial estimation of phenology can be used, but with a small number of field measurements. A second advantage is the possibility of mapping variability of phenology when only one measurement is collected. To our knowledge, such an approach has never been used to estimate grapevine phenology at the vine field scale. Therefore, the aim of this study is to investigate the relevance of an empirical approach based on ancillary data that improves the estimation of the spatial variability of the grapevine phenology at the within-field scale, and (ii) to compare the estimation with classical approaches based on extensive sampling or climatic models.

\section{Materials and methods}




\section{1.- Experimental Fields}

The study was conducted in the Panguilemo Experimental Station of the University of Talca, Maule Valley, Chile. Two fields were chosen, one of cv Cabernet Sauvignon (CS, 1.56 ha; 3522.0' S, 71³5.6' W) and the other of cv Chardonnay $\left(\mathrm{CH}, 1.66 \mathrm{ha} ; 5^{\circ} 21.9^{\prime} \mathrm{S}, 71^{\circ} 35.8^{\prime} \mathrm{W}\right)$. Within each vine field, a regular sampling grid was designed, one with 18 measurement sites $(25 \times 25 \mathrm{~m})$ for cv Cabernet Sauvignon (Fig. 1a) and another one with 19 measurement sites (25x25 m) for cv Chardonnay (Fig. 1b). Each site of the grid was represented by four consecutive vines, located in the same row. For both fields, several specific sites chosen randomly were considered to test the different approaches: a reference site (Fig. 1, in a square) and two sampling sites (Fig. 1, in a circle). More explanations on how these specific sites were defined and used for each field are given in Section 3.

The borders of the fields and sampling sites were geo-referenced with a differential global positioning system receiver (DGPS) (Trimble, Pathfinder ProXRS, Sunnyvale, California, USA) and stored as easting and northing coordinates (Datum WGS84, UTM projection, Zone 19S) for the purposes of mapping.

\section{2.- Field measurements}

This experiment considered 4 seasons for the cv Cabernet Sauvignon (seasons 2009-10, 2010-11, 2011-12 and 2012-13) and 2 seasons for the cv Chardonnay (seasons 2011-12 and 2012-13). Phenological observations were estimated using the Eichhorn and Lorenz phenological scale as modified by Coombe (Coombe 1995). The phenological scale assigns a number to each phenological stage (expressed in units of Phenological Scale, PS). Phenological measurements were performed from pre-budburst (PS 2 to 3 ) to post-veraison (PS 36), every 5-7 days from 30 shoots chosen systematically among the 4 vines of a site on the grid. For each site, the date of occurrence of key phenological stages were calculated by linear interpolation of the recorded values. The following phenological stages were considered:

a) Budburst: Green tip, first leaf tissue visible (PS 4).

b) Flowering: 50\% caps off (PS 23).

c) Veraison: Berry softening and colouring begins (PS 35).

For a given site, a phenological stage was considered to be reached when over $50 \%$ of the shoots were observed.

Climate data (air temperature) were collected using an automatic weather station (Adcon Telemetric, A730, Klosterneuburg, Austria) installed under reference conditions at $300 \mathrm{~m}$ from the vineyards. Air temperature was measured at 15 -min intervals. 

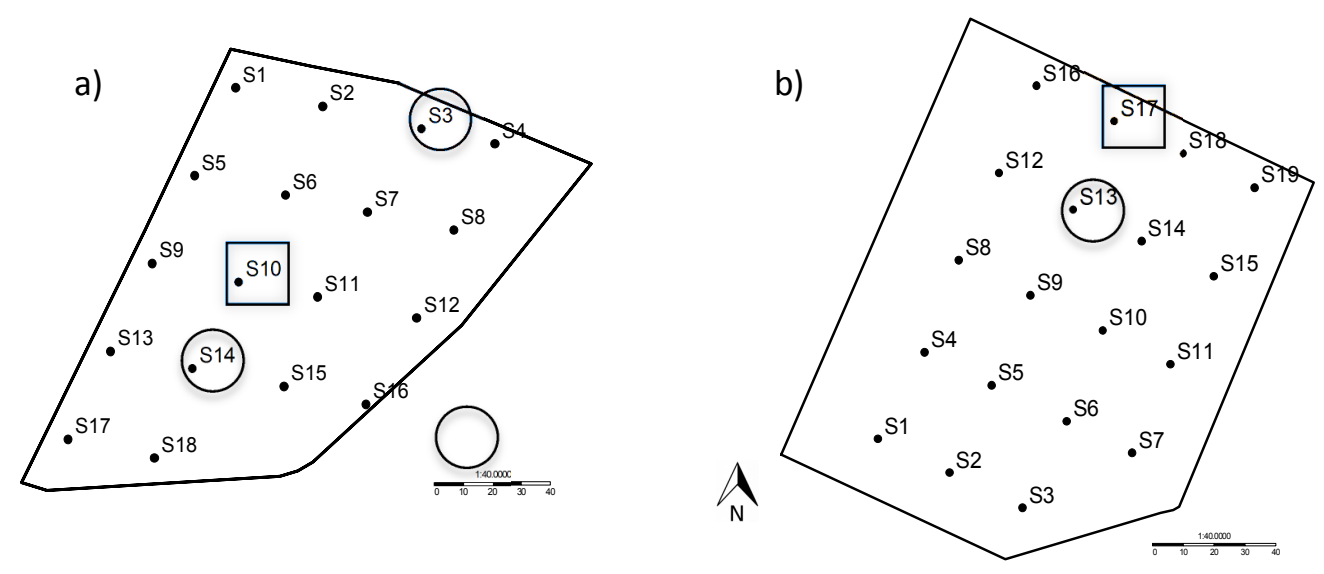

Figure 1 Measurement grids used in the experiments. a) 18 sampling sites for cv Cabernet Sauvignon and b) 19 sampling sites for cv Chardonnay. $\mathrm{S}_{\mathrm{i}}$ represents the sampling site number i. Random sites used for the classical approach are shown in a circle, while the reference site chosen randomly for the spatial model is shown in a square.

\section{3.- Model approaches}

\subsection{Climatic model}

There are different climatic models to predict grapevine phenology (Chuine et al. 2013). In this study, the Growing Degree Day model (GDD model) was used to predict the date of occurrence of the phenological stages of budburst, flowering and veraison. The GDD model is based on the classical thermal time concept (Bonhomme 2000). It assumes that the date of occurrence $t_{s}$ of a phenological stage (DatePS in day of the year, DOY) occurs when a critical state of forcing $S_{f}$, defined as a sum of growing degree days from a starting date $t_{0}$, reaches a particular value $F^{*}$ (Eq. 1) (García de Cortázar-Atauri et al. 2009; Parker et al. 2013).

\section{(Eq. 1)}

The state of forcing is described as a daily sum of the rate of forcing, (Eq. 2) which starts at $t_{0}, x_{t}$ is the daily mean temperature and $T_{b}$ corresponds to a base temperature above which the thermal summation is calculated.

(Eq. 2) 
This approach has three parameters: $t_{0}, F^{*}$ and $T b$. Values for the above parameters are largely provided in the available literature. Table 1 shows the specific values used in this study for the different phenological stages for both cultivars studied.

Table 1 Parameter values considered for the climatic model (GDD model, Eq. 1 and 2)

\begin{tabular}{|c|c|c|c|c|c|}
\hline \multirow[b]{2}{*}{ Cultivar } & \multicolumn{5}{|c|}{ Parameter values } \\
\hline & $\begin{array}{c}\text { Phenological stage } \\
\text { (PS) }\end{array}$ & $\begin{array}{c}\text { Starting date of } \\
\text { GDD } \\
\text { acumulation, } \\
\left(t_{0}\right) \text { (Days of the } \\
\text { year, DOY) }\end{array}$ & $\begin{array}{c}\text { Base } \\
\text { Temperature } \\
\left(t_{b}\right)\left({ }^{\circ} \mathrm{C}\right)\end{array}$ & $\boldsymbol{F}^{*}$ & Source \\
\hline \multirow[t]{2}{*}{ CS } & Flowering (PS 23) & 241 & 0 & 1299.0 & \multirow[b]{2}{*}{ Parker et al. (2013) } \\
\hline & Veraison (PS 35) & 241 & 0 & 2689.0 & \\
\hline \multirow[t]{2}{*}{$\mathbf{C H}$} & Flowering (PS 23) & 241 & 0 & 1217.0 & \multirow[b]{2}{*}{ Parker et al. (2013) } \\
\hline & Veraison (PS 35) & 241 & 0 & 2547.0 & \\
\hline
\end{tabular}

CS: Cabernet Sauvignon, CH: Chardonnay

For the calculation of GDD (Eq. 2), air temperature collected by the automatic weather station was used. This calculation provided one estimate of the date of occurrence of each phenological stage for the whole field (for each cultivar). The predicted date by the climatic model (DatePS) corresponds to the days of the year $\left(t_{s}\right)$ when GDD reaches the value $F^{*}$.

Two approaches were considered for applying the climatic model:

a.- Climatic Model Direct (Cl-M Direct)

This approach directly uses the climatic model, assuming that the parameter of the model provided by the literature (Table 1) are correct for the conditions found in this study. Therefore, the dates predicted by the model were directly used (DatePS) without considering any adjustments or improvements through a calibration process.

b.- Climatic Model Improved (Cl-M Improved)

To take into account possible differences between field climatic conditions and the weather station located $300 \mathrm{~m}$ away, a correction parameter $C$ was introduced (Eq. 3). C was expressed in days and it was determined by calculating the difference between the average observed date of occurrence of the 
phenological stage (mean of all the sites) and the date predicted by the climatic model for each phenological stage (mean of the first 3 seasons for cv Cabernet Sauvignon and the mean of the first 2 seasons for cv Chardonnay).

(Eq. 3)

Once $\mathrm{C}$ was determined, it was added to the date estimated by the climatic model (GDD Model). The resulting corrected climatic model (Eq. 4) is referred to as the Climatic Model Improved in the rest of this document.

(Eq. 4)

\subsection{Classical Sampling}

Classical sampling of the phenological stages corresponded to visual observations made by vine growers. To perform these observations, phenological scales were used, such as the Eichhorn and Lorenz phenological scale as modified by Coombe (Coombe 1995). Usually, the vine grower performs a few measurements of the grapevine phenology, on 2 or 3 sites per field depending on the size of the field. These measurements are typically distributed over the field without necessarily considering spatial variability. The average of these measurements is then calculated and used to create a whole field measurement. Two approaches were considered in this study, differing in the number of sites considered for estimating the average date of occurrence of the phenological stage:

a) Classical Sampling All Sites (Cl-S All Sites): The average date of occurrence of each phenological stage considering all sampling sites (18 sites). Note that this approach is cumbersome since a lot of sites have to be monitored manually for the complete vegetative cycle. From a practical standpoint, this approach maybe unrealistic if it has to be performed regularly. However in this work, it was considered as a reference since it provides the best possible estimation of the phenology of the fields as well as their spatial variability.

b) Classical Sampling 2 Sites (Cl-S 2 Sites): The average date of occurrence of each phenological stage considering 2 random sampling sites as if performed by a vine grower. In this case, the sampled sites were randomly selected from each field. For cv Cabernet Sauvignon, sites 3 and 14 were chosen (Fig 1a, in a circle), while for the cv Chardonnay, sites 6 and 13 were chosen (Fig 1b, in a circle).

3.3 Spatial models 
This approach assumes a temporal stability of the within-field variability (TSWF) of grapevine phenology (Verdugo-Vásquez et al. 2016). In the case of a TSWF, the spatial model considers the use of ancillary data of phenology to model the spatial structure of the fields. Once modeled, the spatial structure is updated with a phenological measurement carried out at a single reference site, which makes it possible to estimate the phenology on all the sites in the field. This is a spatial extrapolation approach and is described by Acevedo-Opazo et al. (2010).

In the present work, the spatial model was used to propose an estimate of the date of occurrence of the phenological stage $P S$ on site $s_{i}$ () using observations collected from a reference site ( $\operatorname{DatePS}\left(s_{\text {ref }}\right)$ ). The spatial model is used to extrapolate the relative differences of the date of occurrence of $P S$ between each site $s_{i}$ and the reference site $\left(s_{r e f}\right)$. This approach predicts the precocity of $P S$ by considering the delay or the advance with respect to $s_{r e}$. Several functions may be considered for modeling this delay or advance. As a first attempt, a simple linear function was considered (Eq. 5). Therefore, as indicated in Eq. 5, the spatial model is based on a collection of linear coefficients :

\section{(Eq. 5)}

Where

$\operatorname{DatePS}\left(s_{i}\right)=$ Date of occurrence of phenological stage $P S$ at site $s_{i}$.

$\operatorname{DatePS}\left(s_{\text {ref }}\right)=$ Date of occurrence of phenological stage $P S$ at a reference site $s_{r e f}$.

$P S$ : corresponds to the phenological stage of budburst, flowering or veraison.

Calibration of the spatial model is based on the the estimation of. This calibration requires ancillary data (historical information) of the date of occurrence of the phenological stage at the vine field scale. In the rest of the study, the collection of the coefficient will be called vector $a$. Two approaches were considered for its calibration:

a) Spatial Model All PS (S-M All PS): assumes that the spatial model is stable over time for all phenological stages. This means that delays or advances remain constant whichever the phenological stage under consideration. In the S-M All PS calibration, historical information from all stages of phenology are considered (budburst, flowering and veraison) and therefore a single value of vector $a$ is obtained for each site.

b) Spatial Model Each PS (S-M Each PS): this approach assumes that the delays or advances in phenological stages may differ depending on the phenological stage under consideration. In the S- 
M Each PS calibration, historical information of phenology are considered separately for each phenological stage and therefore, a value of vector $a$ is obtained for each site and each phenological stage (budburst, flowering and veraison).

In a first approach, the reference site for each cultivar was selected at random. For cv Cabernet Sauvignon and cv Chardonnay, respectively sites 10 (Fig. 1a, in a frame) and 17 (Fig. 1b, in a frame) were chosen. Vector $a$ coefficients were determined using a classical least-squares method as described by AcevedoOpazo et al. (2010). A summary of all the models used in this study along with their characteristics is presented Table 2 .

Table 2 Summary of the different models used and their characteristics.

\begin{tabular}{|c|c|c|c|c|c|c|}
\hline $\begin{array}{l}\text { Type of } \\
\text { model }\end{array}$ & Model name & $\begin{array}{c}\text { Use of } \\
\text { historical } \\
\text { information }\end{array}$ & $\begin{array}{c}\text { Use of } \\
\text { phenology } \\
\text { observations }\end{array}$ & $\begin{array}{c}\text { Use of a } \\
\text { reference site }\end{array}$ & $\begin{array}{c}\text { Use of climatic } \\
\text { data }\end{array}$ & $\begin{array}{c}\text { Characterization } \\
\text { of spatial } \\
\text { variability }\end{array}$ \\
\hline \multirow[b]{2}{*}{ Climatic } & \multirow{2}{*}{$\begin{array}{l}\text { Cl- M Direct } \\
\text { Cl-M } \\
\text { Improved }\end{array}$} & No & No & No & Yes & No \\
\hline & & Yes & Yes & No & Yes & No \\
\hline Classical & Cl-S All sites & No & Yes & No & No & No \\
\hline Sampling & Cl-S 2 sites & No & Yes & No & No & No \\
\hline \multirow{2}{*}{ Spatial } & S-M All PS & Yes & Yes & Yes & No & Yes \\
\hline & S-M Each PS & Yes & Yes & Yes & No & Yes \\
\hline
\end{tabular}

\section{4.- Calibration and validation of the models}

Depending on the field and the available data, the model calibration process varied slightly. For cv Cabernet Sauvignon, the data from the first 3 seasons were used to calibrate the model, while for cv Chardonnay the data from the 2 available seasons were used. The validation process was performed only with cv Cabernet Sauvignon using the fourth season (2012-2013) data. This procedure is followed to evaluate the performance of the model prediction with data that is different to that used in the calibration process. For both calibration and validation processes, the root mean square error (RMSE, Eq. 6) was used to characterize the phenological stage estimation accuracy of each model.

$R M S E=\sqrt{\frac{\sum_{i=1}^{n}\left(\text { DatePS }_{\text {sim }}-\text { DatePS }_{\text {obs }}\right)^{2}}{n}}$

Where DatePS $S_{\text {sim }}$ is the estimated date of occurrence of the phenological stage; DatePS $S_{\text {obs }}$ corresponds to the observed date of occurrence of the phenological stage and $n$ is the number of observations. The RMSE was estimated for each phenological stage separately. 
Additionally, for both cultivars, differences (expressed in days) between the observed date of occurrence of the phenological stages and the mean date estimated through the Cl-S All Sites model were calculated for each site on the sampling grid using data from the calibration process. These differences were used to show potential patterns of spatial error.

\section{5.- Sensitivity analysis}

As several approaches rely on the selection of specific sites to perform estimations, sensitivity analysis was performed to determine the impact of this choice. This is an issue with two approaches: (i) the Cl-S 2 Sites where estimation relies on the observations collected from two sampling sites and (ii) the S-M (All PS and Each PS) where estimation is based on the extrapolation of the phenological stage that was observed at a reference site. To estimate the potential impact of the choice of these sites, two different methodologies were used:

- For the Cl-S 2 Sites model, all the possible pairs of site combinations were used to estimate the field average value for the date of occurrence of each phenological stage. This was performed with the 2012-13 season data.

- For the S-M, the 18 sample sites were tested as the possible reference site. The analysis was performed for each phenological stage separately and all combined.

The sensitivity analysis considered the probability of obtaining a root mean square error (RMSE) lower than a threshold value (expressed in days). This analysis was performed only on the cv CS.

\section{6.- Time (hours) spent on implementation of each proposed model}

In order to assess practical constraints (time) required for each approach, the time spent on the implementation of each approach was estimated. For that purpose, only the time for field measurements (phenology observations) on the different sites was taken into account, considering the following assumptions:

(i) Field measurements were performed by an experienced technical person (skilled labor).

(ii) Time required to perform an observation at a sample site was 0.17 hours (10 minutes) and this time was assumed constant for the different phenological stages. 
(iii) The date of occurrence of the phenological stages was calculated by linear interpolation of the observed data at two different times. Therefore, 2 measurement dates were used for a reliable estimation of each phenological stage.

(iv) The time spent to collect and process the weather data was not considered (value $=0$ hours).

Using the above assumptions, the time required for each approach was estimated for the calibration of both cultivars. In addition, to estimate the time spent on each approach for commercial use (once calibration of models is effective), the time spent other the season of use was considered on the cv CS.

\section{7.- Mapping}

Maps of the vector $a$ (Spatial model) and spatial errors were generated to visualize the spatial distribution of the error. The interpolation method used in this study was based on a deterministic function (inverse distance weighting). Vector $a$ and spatial errors were mapped to $33 \%$ quantiles for each phenological stage using the 3D-field software (version 2.9.0.0., Copyrigtht 1998-2007, Vladimir Galouchko, Russia).

\section{RESULTS}

\section{Within-field variability of phenology}

Table 3 presents the observed variability of dates of occurrence for the different phenological stages (budburst, flowering and veraison) at the within-field scale for both cultivars. The magnitude of variation (MV) varies between 4 to 12 days for cv Cabernet Sauvignon and 4 to 9 days for cv Chardonnay. For both cultivars, the phenological stage that presented the highest MV corresponded to veraison. With respect to standard deviation (SD), for both cultivars and all phenological stages, SD values were less than 4 days. These preliminary results show that the within-field variability in the date of occurrence of key phenological stages is significant and therefore, if the variability is spatially structured, the planning of operations based on the mean date of occurrence may be inappropriate for a large part of the field.

Table 3 Variability of the phenology at the within-field scale observed for both cultivars over the seasons (values expressed in days).

\begin{tabular}{|c|c|c|c|c|c|c|c|c|c|c|c|c|c|c|c|c|}
\hline \multirow[b]{2}{*}{$\mathrm{cv}$} & \multirow[b]{2}{*}{ Season } & \multicolumn{5}{|c|}{ Budburst } & \multicolumn{5}{|c|}{ Flowering } & \multicolumn{5}{|c|}{ Veraison } \\
\hline & & $\begin{array}{c}\text { Mean } \\
\text { (DOY) }\end{array}$ & SD & Min & Max & MV & $\begin{array}{l}\text { Mean } \\
\text { (DOY) }\end{array}$ & $\mathrm{SD}$ & Min & Max & MV & $\begin{array}{c}\text { Mean } \\
\text { (DOY) }\end{array}$ & SD & Min & Max & MV \\
\hline \multirow{2}{*}{$\mathrm{CS}$} & $2009-10$ & 272 & 2.0 & 270 & 277 & 7 & 338 & 2.6 & 335 & 343 & 8 & 37 & 2.3 & 34 & 40 & 6 \\
\hline & $2010-11$ & 275 & 1.4 & 274 & 278 & 4 & 329 & 2.5 & 327 & 336 & 9 & 26 & 2.1 & 23 & 31 & 8 \\
\hline
\end{tabular}




\begin{tabular}{ccccccccccccccccc}
\hline & $2011-12$ & 266 & 2.4 & 264 & 272 & 8 & 324 & 2.6 & 321 & 331 & 10 & 23 & 2.5 & 21 & 30 & 9 \\
& $2012-13$ & 270 & 2.5 & 268 & 276 & 8 & 327 & 1.7 & 324 & 331 & 7 & 29 & 3.4 & 23 & 35 & 12 \\
& $2011-12$ & 264 & 2.6 & 259 & 267 & 8 & 320 & 1.0 & 318 & 322 & 4 & 19 & 2.0 & 15 & 22 & 7 \\
$\mathrm{CH}$ & $2012-13$ & 263 & 2.5 & 258 & 265 & 7 & 323 & 2.3 & 319 & 325 & 6 & 22 & 3.5 & 17 & 26 & 9
\end{tabular}

CS: Cabernet Sauvignon, CH: Chardonnay, SD: Standard deviation, Min: Minimum, Max: Maximum, MV: Magnitude of Variation. DOY: Day of the year.

\section{Model calibration}

The errors, expressed as RMSE, calculated for the different models are shown in Table 4. For both cultivars, the magnitude of variation of RMSE values range from 1 to 13 days, showing a high variability in the quality of estimation according to the model used. In relation to the estimation of key phenological stages, veraison is the phenological stage that systematically presents the highest RMSE values, whatever the model used and for both cultivars. As expected with results of within field variability (Table 3), veraison is the phenological stage which is the most difficult to estimate precisely, whereas budburst and flowering show very similar behaviour.

On average (last rows in Table 4), when results are ordered from the lowest to the highest RMSE, the order of the models is generally always the same: The Spatial Models (S-M All PS or Each PS) always shows the lowest RMSE, followed by the Classical-Sampling (Cl-S -All Sites and 2 Sites). The Climatic models (Cl-M Direct and Improved) shows the highest RMSE systematically. The adjustment of Cl-M (Improved) substantially reduces RMSE compared to the results observed with the Cl-M Direct. The higher RMSE values observed for the Cl-M (Direct and Improved) may be explained by predictions calculated with indirect information (air temperature) used in the associated model. Two main sources of error may result from an approach based on climatic data. Firstly, climatic data are provided by a weather station that is not located in the field but nearby, therefore climatic data may differ from that experienced by the field under study, secondly the model itself may be too general and may not be well adapted to the peculiarities of the fields under study. As shown in Table 4 with Cl-S and S-M (and to a lesser extent with Cl-M Improved), the use of direct phenological observations within the field improves significantly the quality of estimations.

The RMSE values of the Cl-S models (All Sites and 2 Sites) are similar and lower than 3 days for both cultivars (Table 4). For cv Cabernet Sauvignon, both S-M (All PS and Each PS) generate the lowest RMSE. However, for cv Chardonnay, only the S-M Each PS generate lower RMSE than those obtained with the Classical Sampling models (All Sites and 2 Sites). 
These results show that there are significant differences in the quality of the estimation obtained with the different models. To explain these differences, a comparison between the different models, using the Cl-S (All Sites) as a reference was carried out and is presented in the following section.

Table 4 Errors (RMSE) obtained from the different models for both cultivars (calibration process).

\begin{tabular}{|c|c|c|c|c|c|c|c|}
\hline \multirow[b]{3}{*}{ Cultivar } & \multirow[b]{3}{*}{$\begin{array}{l}\text { Phenological } \\
\text { stage }\end{array}$} & \multicolumn{6}{|c|}{ RMSE (Days) } \\
\hline & & \multicolumn{2}{|c|}{ Climatic (Cl-M) } & \multicolumn{2}{|c|}{$\begin{array}{l}\text { Classical Sampling } \\
\text { (Cl-S })\end{array}$} & \multicolumn{2}{|c|}{ Spatial (S-M) } \\
\hline & & Direct & Improved & All Sites & 2 Sites & All PS & Each PS \\
\hline \multirow[t]{3}{*}{$\mathrm{CS}$} & Budburst & 7.2 & 5.0 & 2.0 & 2.1 & 1.3 & 1.0 \\
\hline & Flowering & 7.0 & 3.5 & 2.5 & 2.7 & 1.3 & 1.1 \\
\hline & Veraison & 13.0 & 3.5 & 2.2 & 2.3 & 2.9 & 2.4 \\
\hline \multirow[t]{3}{*}{$\mathrm{CH}$} & Budburst & 13.1 & 3.1 & 2.4 & 2.5 & 1.1 & 0.2 \\
\hline & Flowering & 7.5 & 2.4 & 1.8 & 1.9 & 1.9 & 1.6 \\
\hline & Veraison & 9.7 & 3.0 & 2.7 & 2.7 & 4.2 & 1.2 \\
\hline $\mathrm{CS}$ & & 9.0 & 4.0 & 2.2 & 2.4 & 1.8 & 1.5 \\
\hline $\mathrm{CH}$ & Mean of all PS & 10.1 & 2.9 & 2.3 & 2.4 & 2.4 & 1.0 \\
\hline
\end{tabular}

*PS: Phenological stages. CS: Cabernet Sauvignon, CH: Chardonnay.

Comparison between the Classical Sampling model (All Sites) and the Climate model Direct.

Figure 2 shows that there is a bias between the average date of occurrence of the phenological stage and estimation provided by Climatic Model when compared to estimations based on sampling (Cl-S All Sites and 2 sites). This bias explains the reason for the high RMSE observed for this method (Table 4). Confirming previous results, the dates of occurrence estimated by both Cl-S are very similar. Note, however, that the distance (in days) between both methods changes with the phenological stage, showing that an estimation based on only 2 sites may adversely affect the quality of the outputs. Figure 2 shows also that the Cl-S (All Sites and 2 Sites) are not able to characterize the variability of the date of occurrence of the phenological stages at the within-field scale. Dispersion, caused by the within-field variability, is the main variability at this scale and explains a large proportion of the RMSE for both these approach. 

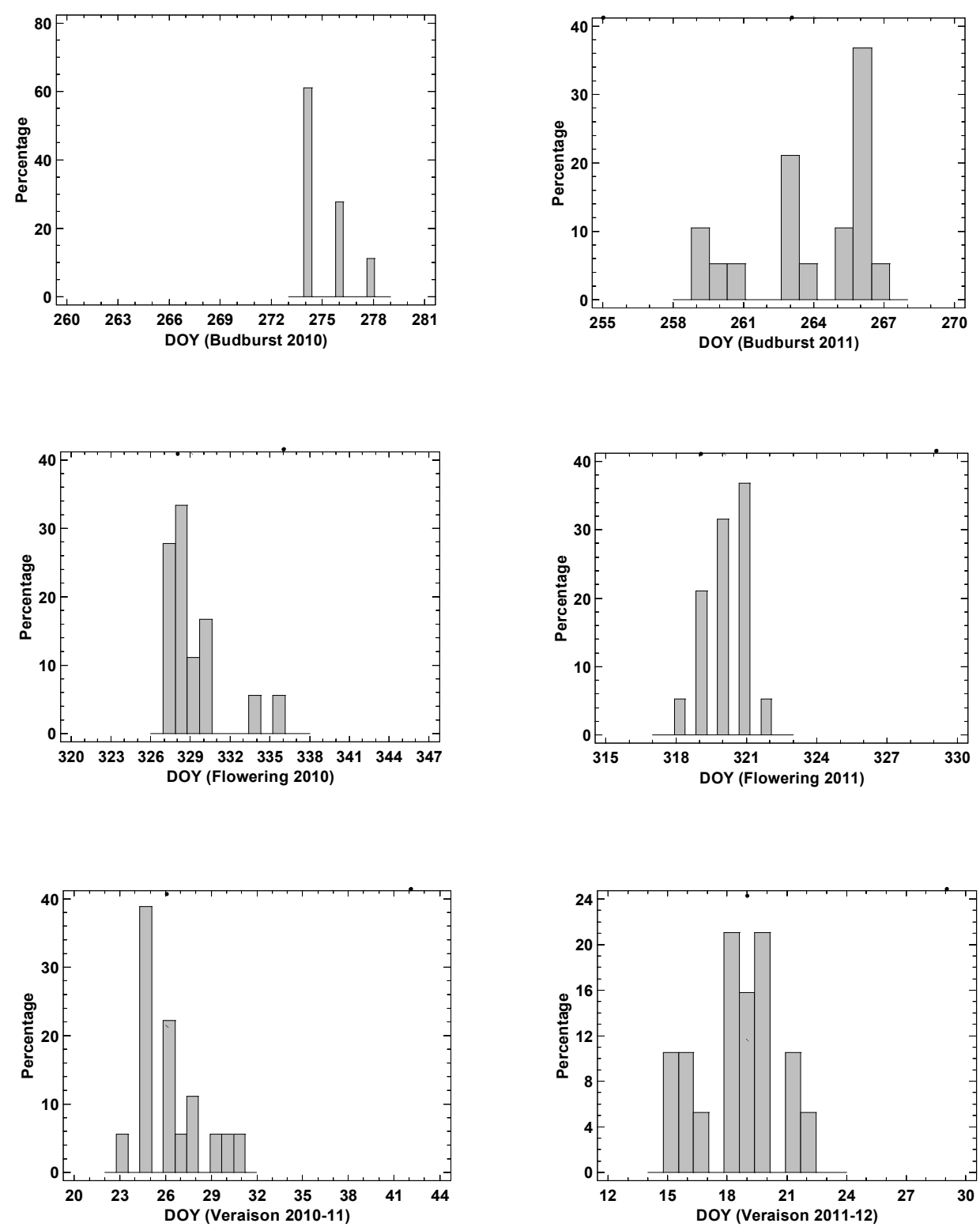

Figure 2 Histogram of the date of occurrence (DOY) of budburst (a), flowering (b) and veraison (c), for cv Cabernet Sauvignon (seasons 2010-11) and cv Chardonnay (season 2011-12) for all the sites. Black lines: date of occurrence of phenological stage observed (mean of all sites); Dotted lines: observed date of occurrence of phenological stage (mean 2 sites); Dashed lines: estimated date of occurrence by the Climatic model (Direct) for the whole field.

Table 5 confirms the results observed in Figure 2 for all the seasons studied. A bias is systematically observed between the observed date of occurrence (Cl-S) and the date estimated by $\mathrm{Cl}-\mathrm{M}$ Direct over all 
the seasons. For budburst, this difference is positive in most of the cases showing that Climatic model underestimates the date of occurrence of this phenological stage. For flowering and veraison, differences are negative, indicating an overestimation of the date of occurrence for both of these phenological stages with the Climatic model. This result proves the necessity to introduce a correction coefficient (Eq. 3 and Eq. 4) to account for site-specific set up of the climatic model. Results shown in Table 5 highlight a significant limit the climatic model when applied without taking into account the specific conditions of the field and/or the relative distance of the field under study from the meteorological station.

Table 5 Date of occurrence of phenological stage (DOY) estimated by the Climatic model Direct (estimated - Est) and by classical sampling of all sites as a reference (observed - Obs). The magnitude of variation represents the largest difference between observed (Obs) and estimated (Est) dates expressed in days and Growing Degree Day (GDD).

\begin{tabular}{|c|c|c|c|c|c|c|c|c|c|c|c|c|c|}
\hline \multirow[b]{3}{*}{$\mathrm{cv}$} & \multirow[b]{3}{*}{ Season } & \multicolumn{4}{|c|}{ Budburst } & \multicolumn{4}{|c|}{ Flowering } & \multicolumn{4}{|c|}{ Veraison } \\
\hline & & \multicolumn{2}{|c|}{ DOY } & \multicolumn{2}{|c|}{$\begin{array}{c}\text { Magnitude of } \\
\text { variation }\end{array}$} & \multicolumn{2}{|c|}{ DOY } & \multicolumn{2}{|c|}{$\begin{array}{c}\text { Magnitude of } \\
\text { variation }\end{array}$} & \multicolumn{2}{|c|}{ DOY } & \multicolumn{2}{|c|}{$\begin{array}{c}\text { Magnitude of } \\
\text { variation }\end{array}$} \\
\hline & & Est & Obs & Days & GDD & Est & Obs & Days & GDD & Est & Obs & Days & GDD \\
\hline \multirow{5}{*}{$\mathrm{CS}$} & $2009-10$ & 263 & 272 & 9 & 51.9 & 341 & 338 & -3 & -51.4 & 46 & 37 & -9 & -176.3 \\
\hline & 2010-11 & 267 & 275 & 8 & 50.6 & 336 & 329 & -7 & -122.4 & 42 & 26 & -16 & -321.4 \\
\hline & & & & & & & & & & & & & \\
\hline & 2011-12 & 267 & 266 & -1 & -8.9 & 333 & 324 & -9 & -164.1 & 36 & 23 & -13 & -264.4 \\
\hline & Mean & 266 & 271 & 5 & 31.2 & 337 & 331 & -6 & -112.6 & 41 & 29 & -13 & -254.0 \\
\hline \multirow{3}{*}{$\mathrm{CH}$} & 2011-12 & 253 & 264 & 11 & 78.7 & 329 & 320 & -9 & -152.5 & 29 & 19 & -10 & -197.9 \\
\hline & $2012-13$ & 248 & 263 & 15 & 97.7 & 328 & 323 & -5 & -84.6 & 30 & 22 & -8 & -171.1 \\
\hline & Mean & 251 & 263 & 13 & 88.2 & 329 & 321 & -7 & -118.5 & 30 & 20 & -9 & -184.5 \\
\hline
\end{tabular}

CS: Cabernet Sauvignon, CH: Chardonnay. DOY: Days of the years.

\section{Comparison between the Classical Sampling model (All Sites) and the Spatial model}

As previously mentioned, the Classical Sampling approach (Cl-S) does not account for the within field variability of the date of occurrence of the phenological stages. Figure 3 shows maps representing the difference between the phenological stage observed on each site and the mean of the field (All Sites). In order to facilitate comparison with Figure 2, the maps shown in Figure 3 where generated for the same seasons. This difference will be called error in the rest of the section. Figure 3 highlights a high spatially organized error for both cultivars. For all the phenological stages and both cultivars, zones in advance 
(negative values) and zones in delay (positive values) are clearly observed. Errors are not randomly distributed over the fields. They correspond to distinct zones which are time stable over the different phenological stages for both cultivars. For cv Cabernet Sauvignon, the southern part of the field (dark gray), systematically presents zones in delay of 3 to 7 days according to the mean of the field (for the 2010-11 season) while for cv Chardonnay, the northern part of the field (light gray), systematically presents zones in advance of 6 to 3 days (for the 2011-12 season). The same patterns were observed for the other seasons studied (data not shown).

Figure 4 shows how the Vector $a$ of the spatial model (S-M All PS) obtained after calibration, account for the spatial distribution of the phenology for both cultivars. Spatial patterns of the vector $a$ are very similar to error maps (Fig. 3), showing the ability of the Vector $a$ to model the delay or the advances in phenology at the within field level. This explains the better results (lower RMSE values) observed with spatial models (Table 4). 
a)

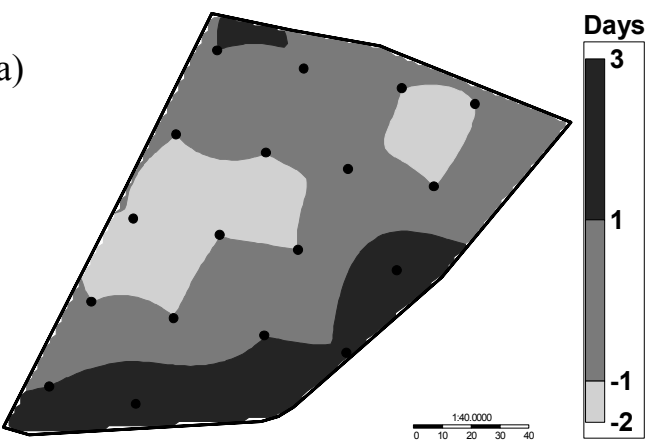

b)
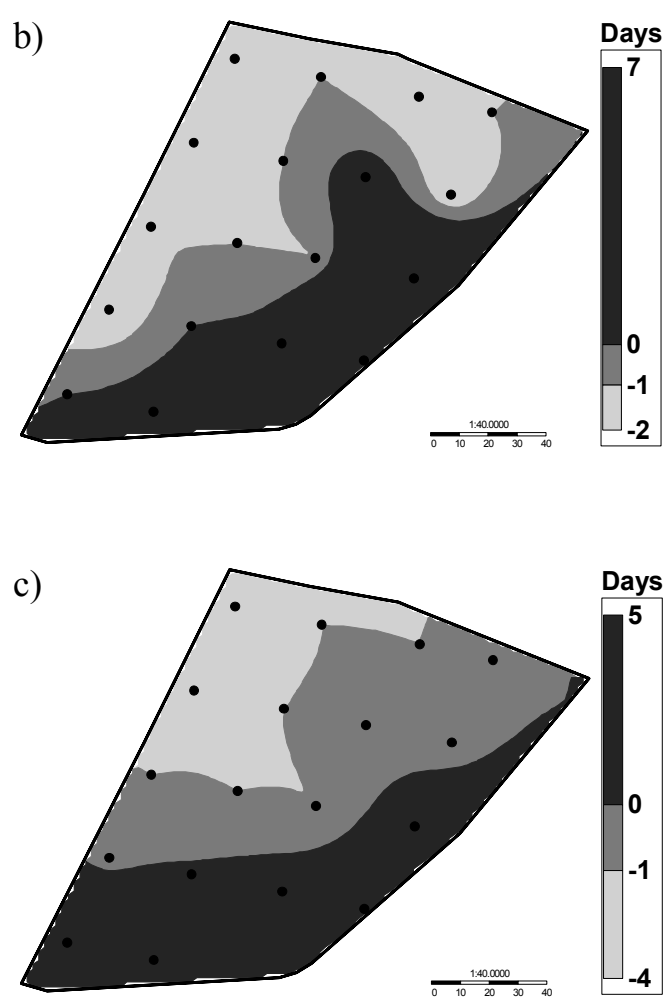

c)

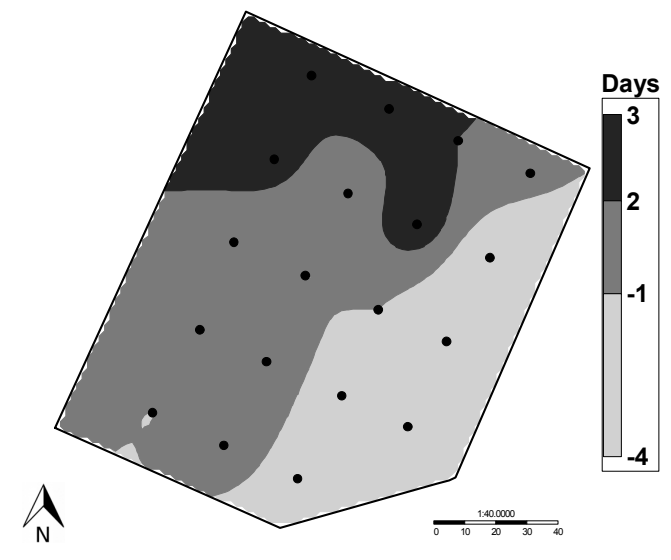

Figure 3 Differences (error) between the observed date of occurrence of phenological stages and the mean date estimated through the Classical Sampling (All Sites) for both cultivars, cv Cabernet Sauvignon (season 2010-11) and cv Chardonnay (season 2010-11). a) Budburst, b) Flowering and c) Veraison. 

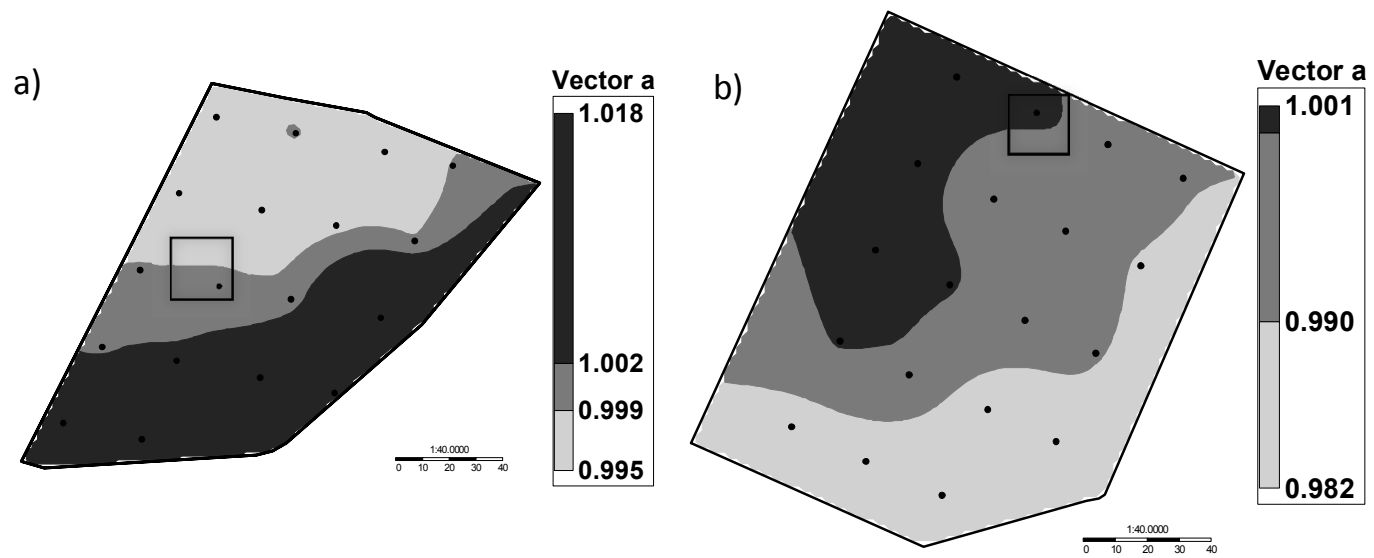

Figure 4 Vector $a$ obtained after the calibration process, cv Cabernet Sauvignon (a) and cv Chardonnay (b) for the spatial model "All PS". Reference site is shown in a square.

\section{Model validation}

Table 6 presents the RMSE values obtained when predicting date of phenology. Recall that strictly speaking, predictions are performed once a model is calibrated and when an independent dataset is used to test the ability of the model to predict values. These conditions apply to all models except for Classical Sampling (Cl-S) which requires observations over the whole field at each date. However, Cl-S has been considered in Table 6 as a reference method since it corresponds to a current approach. Very slight increases in RMSE values were observed when comparing results of calibration to those of validation. Table 6 shows therefore the ability of all models to estimate the date of occurrence of key phenological stages with an independent data set. Validation results highlight the same trends as for calibration. Better predictions (lower RMSE values) are observed for almost all the phenological stages when direct observations are collected in the fields, either with spatial modelling or sampling approaches. The poorest results were observed for models based on climatic data (Cl-M either direct or improved). Veraison remains the phenological stage with the highest RMSE for all the models and flowering the lowest.

In general, spatial models (S-M) and models based on classical sampling (Cl-S) present similar results. Climate based models (Cl-M) provide better predictions for flowering than for budburst and veraison. This aspect may be of interest from a practical point of view, since depending on the expected accuracy, different approaches can be considered in terms of their phenological stage. Note however that a 5 day error on phenology estimation may be sufficient for many applications and that the choice of a more precise estimation method should take into account the practical constraints. These constraints differ greatly from one method to another. For example, the Cl-S requires observations to be made on all sites systematically and the S-M requires a single observation on a reference site (provided that a historical 
database is available for the calibration). More details about operational constraints will be presented in a next section.

Table 6 Errors (RMSE) obtained by the different model approaches (cv Cabernet Sauvignon), validation process.

\begin{tabular}{ccccccc}
\hline & \multicolumn{5}{c}{ RMSE (Days) } \\
& \multicolumn{2}{c}{ Climatic (Cl-M) } & \multicolumn{2}{c}{$\begin{array}{c}\text { Classical Sampling } \\
\text { (Cl-S) }\end{array}$} & \multicolumn{2}{c}{ Spatial* $^{*}$ (S-M) } \\
$\begin{array}{c}\text { Phenological } \\
\text { stage }\end{array}$ & Direct & Improved $^{*}$ & All Sites & 2 Sites & All PS & Each PS \\
\hline Budburst & 8.5 & 4.1 & 2.4 & 3.3 & 2.3 & 2.2 \\
Flowering & 5.1 & 1.9 & 1.6 & 1.7 & 2.0 & 1.9 \\
Veraison & 7.7 & 6.5 & 3.2 & 3.4 & 3.1 & 2.5 \\
All PS (Mean) & 7.1 & 4.2 & 2.4 & 2.8 & 2.5 & 2.2 \\
\hline
\end{tabular}

"Only for Improved and Spatial models is considered as a validation process.

Sensitivity analysis to the selection of the sample sites (Classical Sampling) and the reference site (Spatial model)

The sensitivity analysis was conducted for approaches that rely on the selection of sites of observations (Cl-S -2 Sites and S-M -All PS and Each PS). For the S-M, this is related to the choice of the reference site while for the Cl-S (2 Sites), this is related to the choice of the two sites used to estimate the mean of the field. The sensitivity analysis considered the probability of obtaining a root mean square error (RMSE) lower than a threshold value (expressed in days). For example, the probability of obtaining RMSE values lower than 3 days for the S-M -Each PS, S-M -All PS and Cl-S -2 Sites corresponds to $80 \%, 55 \%$ and $60 \%$, respectively (Fig. 5a). The result of this analysis showed that in general, the different approaches were not significantly affected by the choice observation sites (sample sites or reference site). Since in 95\% of cases, this choice affects the RMSE value by a maximum of 1 day whatever the approach considered (Fig. 5a).

For the phenological stages with low prediction RMSE, i.e. flowering and to a lesser extent budburst, the impact of the choice of the sites of observations is generally low. The probability of observing a RMSE value lower than 3.5 days is approximatively $90 \%$ whatever the considered approach (Figure $5 \mathrm{~b}$ and $5 \mathrm{c}$ ). On the other hand, for veraison, the choice of the site(s) seems more important. Indeed, considering only the Spatial model (All PS), the choice of the reference site leads to a RMSE ranging from less than 2.5 days in $10 \%$ of the cases to less than 4.5 days in $90 \%$ of the cases. 


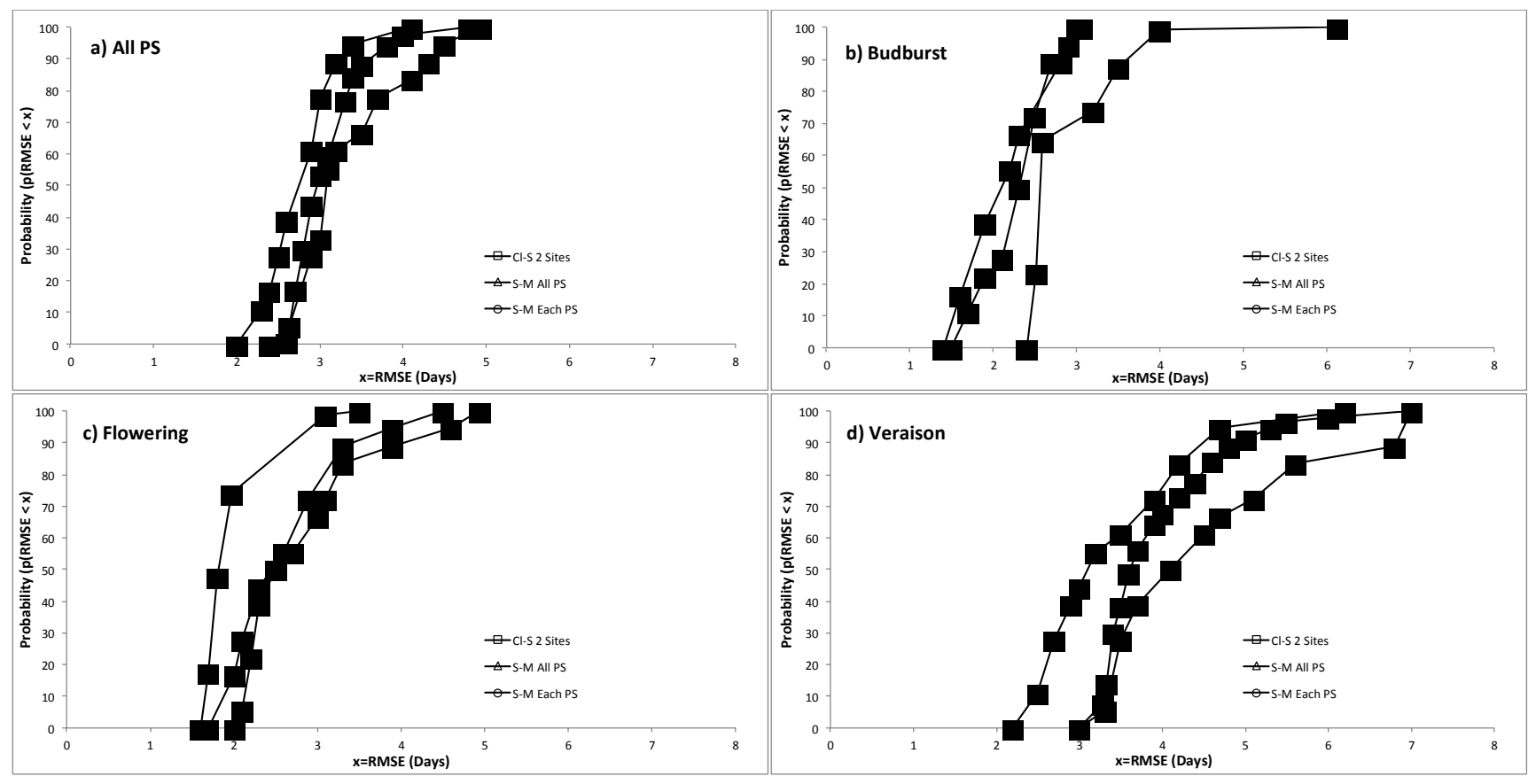

Figure 5 Sensitivity analysis of the choice of reference sites for S-M -All PS and Each PS and the choice of the two sites for the Cl-S -2 Sites, cv Cabernet Sauvignon. Figures represent the probabilities of obtaining a RMSE below a threshold value.

\section{Time spent on implementation of each model approach}

The different approaches proposed in this paper give different results in terms of precision. However, their practical implementation is more or less restrictive. This is why it is important to study the practical limitations, estimated by the time to collect information compared to the precision of the expected results. The calibration and prediction steps were considered separately. The prediction corresponds to a commercial use, that is, once the model is calibrated. The distinction between prediction and calibration is important since a precise and non-constraining approach for commercial use may justify a significant effort for its calibration since this last step will be short in time ( 2 years or 3 years) compared to the period of use in commercial conditions.

Regarding calibration, Figure 6a clearly shows that Cl-M Direct behaves differently to all other approaches (Cl-M Improved, Cl-S All sites and S-M) requiring a large and comprehensive number of observations. The Direct method does not require any field observations and is therefore straightforward to implement but generates an imprecise model whereas the models based on field observations show the opposite behaviour. Note however the particular position of the Cl-S (2 sites) model which makes it possible to obtain accurate estimations with limited implementation constraints. 
For commercial use (Fig. 6b), it was observed that for all the models, the time spent is lower than that observed in the calibration process. This figure clearly shows that the Cl-S (All sites) method that requires sampling all sites of the field is quite different to the other approaches. From a practical and commercial point of view, Cl-S (All sites) seems to be unrealistic because it would still require a lot of time for commercial use while the gain in the quality of the estimation is small compared to the other approaches. All other methods present very similar implementation times (less than 1 hour). Therefore, the choice of one method or another may be based on the quality of the estimation that they provide. From this point of view, there is a clear difference between the methods based on climate information (Cl-M Direct and Improved) and that based on observations (Cl-S 2 Sites and S-M All PS and Each PS). If the expected precision is less than 3 days, methods based on observations are advisable, knowing that no significant differences exist between them. Finally, it is also of interest that the Cl-S (2 Sites) presents a high precision compared to Spatial Models (All PS and Each PS), which are the most accurate (lowest RMSE values).

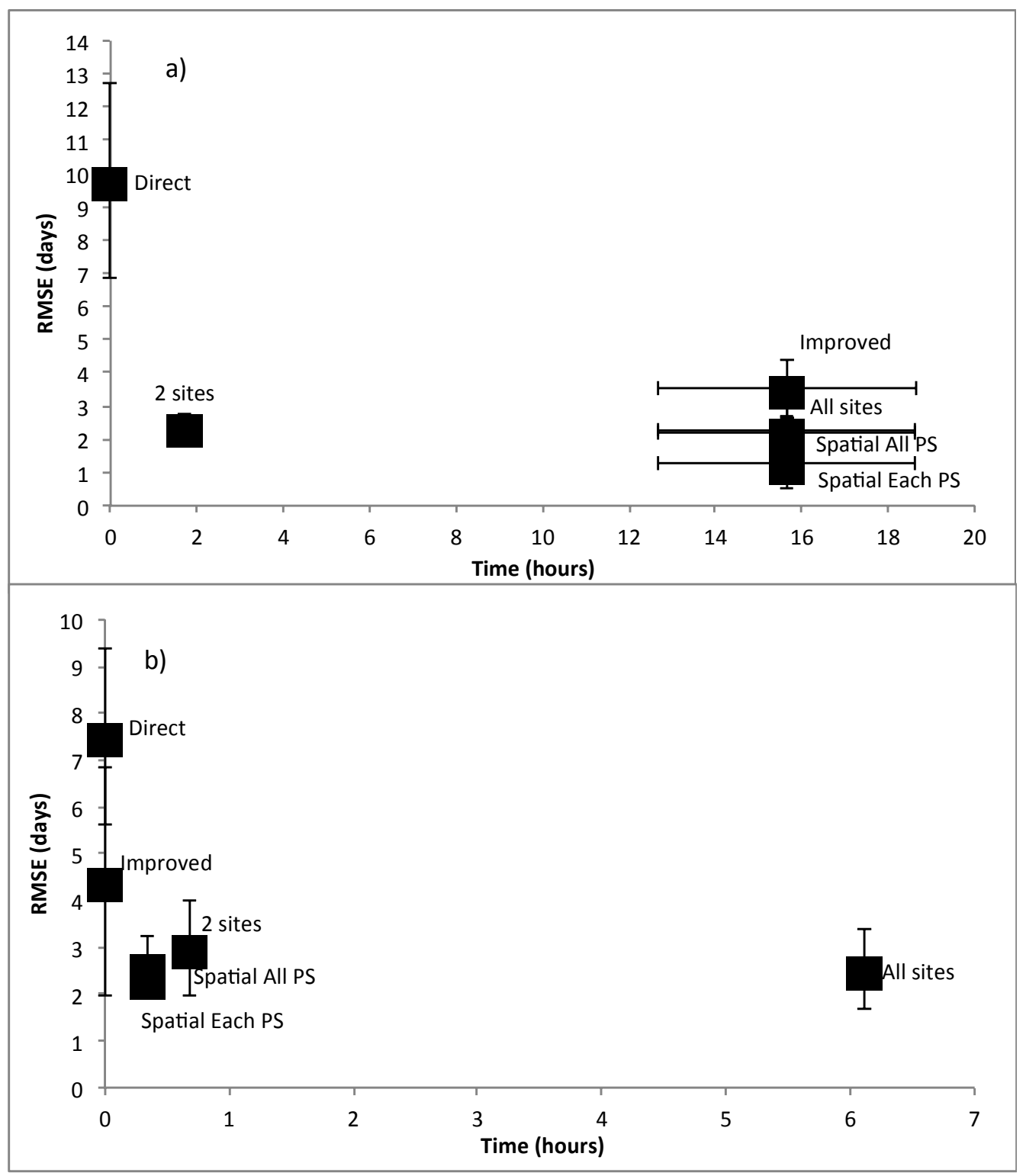


Figure 6 RMSE (days) obtained with the different approaches versus the time (hours) spent on their implementation (mean of all phenological stages). a) Calibration process (both cultivars) and b) Commercial use (only for cv Cabernet Sauvignon). The bars represent the standard deviation accounting for the dispersion observed between phenological stages.

\section{DISCUSSION}

This study presents an empirical approach (spatial model) for estimating the grapevine phenology at the within-field scale. The interest of this approach is its ability to account for the within field variability while minimizing the constraints of sampling and data collection by taking into account only one observation taken from a reference site. The predictions of the proposed approach are good enough to be used as a decision support for vineyard management, since the RMSE values of prediction are lower or similar to those observed by the best possible approach (Cl-S, All Sites), within an interval of 2 days of errors.

The spatial model provides the best estimation when compared to a large range of classical approaches based either on extensive sampling or on climatic data. Note that the methodology used was derived directly from the one proposed by Acevedo-Opazo et al. (2010). It is based on a collection of linear coefficients and in this work a slight modification was proposed to adapt the coefficients to the phenological stage (S-M Each PS). This modification gave the best prediction results compared to a more straightforward application of the spatial model (S-M All PS) as proposed by Acevedo-Opazo et al. (2010), showing that other functions to model the relationship between the reference site and the other sitez of the field may be considered to improve the predictions.

\section{Practical aspects}

One significant limit of spatial models is the high time required for their calibration. However, once calibrated, their commercial use requires less time than the other approaches for a better prediction result. Further research should focus on the optimization of the calibration process of these spatial models or the use of other spatial information, this last point will be discussed later in the discussion. From a commercial point of view, the interest of the spatial model may be limited, especially when the spatial variability of phenology is low. In this case our study shows that a more traditional method of sampling (Cl-S, 2 Sites) may be the most relevant approach, becoming a real alternative for the vine-growers. 
Climatic models are quick and easy to implement. Our study shows that they may present some significant limitations related to their inability to account for the specific conditions of the field and the spatial variability of the field. This study proposed two improvements to tackle these issues: i) the introduction of a local coefficient of correction (Cl-M Improved) that takes into account the differences between the climatic conditions measured by the weather station and the climatic conditions of the plant. This approach is simple and can be tested in other conditions (largest distance between the weather station and the field, highest altitude difference, etc.). This improvement requires a calibration step as well as the availability of historical data. ii) Install the weather station or temperature sensor as close as possible to the vineyard, as proposed by Matese et al. (2014). However, this solution has certain uncertainties associated with the cost, location and number of sensors, already mentioned above. Generally, the spatial aspects are less frequently considered, but they have significant effects at different spatial scales. For example, in the Cl-M Improved, the incorporation of a coefficient that takes into account the differences between the temperatures measured by the weather station and the microclimate of the plant, considerably improved the estimation of grapevine phenology. Therefore, the knowledge of spatial variability (elevation, distance to the sea, etc.) is a key component to consider to improve the estimation of grapevine phenology at all spatial scales, whether at the vineyard, field or within-field scale.

\section{Improvements and perspectives of the Spatial models}

This study shows that the use of the spatial models may be a realistic opportunity when phenological stages have to be known with the highest possible accuracy. Given the results in this work, this is one relevant way to provide growers with knowledge of within field zones that present advance or delay in precocity with respect to the reference site. However, the model calibration requires a significant historical database which may present a significant drawback for commercial vineyards. As has been proposed for similar approaches based on spatial extrapolation, the use of high resolution auxiliary information easy to measure at a high spatial resolution and at a lost may constitute a an opportunity. Verdugo-Vásquez et al. (2016) suggested that the spatial variability of stable environmental factors (soil characteristics, slope, soil texture, etc.) would produce differences in the microclimate of the field, which affect the phenology, as well as being able to explain the observed spatial variability. Therefore, characterization of environmental factors at a high spatial resolution could be used as auxiliary information to calibrate the spatial models. Such approaches are common in precision agriculture and well known information like indices derived from airborne imagery, soil electrical conductivity measurements or manual medium-spatial resolution measurements, such as shoot length and exposed leaf area (Acevedo-Opazo et al. 2010), could also be used to zone vineyards according to grapevine phenology and simplify the development and the calibration of the spatial models. 
Finally, it is important to note that the different approaches presented in this study may be complementary. For example, field observations (Cl-S) can be used to calibrate climatic models of phenology, improving the results obtained. On the other hand, the combination of the climatic model of phenology and the Spatial model would allow the prediction of the date of occurrence of the grapevine phenology by taking into account the spatial variability of the field. For this, the date of occurrence predicted by the climatic model could also be incorporated into the spatial model as the reference site measurement, which could then be used to extrapolate the estimated date of occurrence to the entire field. This last approach has not been tested for grapevine phenology.

\section{CONCLUSIONS}

This study showed that empirical spatial models are a potential method for estimating the key phenological stages of grapevines at the within field level. When compared to more classical methodologies, spatial models present the best accuracy and are a suitable alternative when highly accurate estimates of phenology (less than 3 days) are required at the within field level. This study focused on a first step towards modeling the spatial variability of grapevine phenology at the within-field scale. To be fully operational it still requires many improvements that will be investigated in future research: i) the use of high spatial resolution auxiliary data to decrease practical constraints related to the calibration of the model, ii) the combination of both a climatic model and a spatial model to perform prediction of grapevine phenology in both time and space.

\section{ACKNOWLEDGEMENTS}

The authors of this paper wish to thank National CONICYT Doctoral Fellowship 2013 Nº21130504 and PIEI Program of Agricultural Adaptation to Climate Change funded by the University of Talca.

\section{REFERENCES}

Acevedo-Opazo, C., Tisseyre, B., Ojeda, H., \& Guillaume, S. (2010). Spatial extrapolation of the vine (Vitis vinifera L.) water status: a first step towards a spatial prediction model. Irrigation Science, 28(2), 143-155. https://doi.org/10.1007/s00271-009-0170-3

Acevedo-Opazo, C., Tisseyre, B., Taylor, J. a., Ojeda, H., \& Guillaume, S. (2010). A model for the spatial prediction of water status in vines (Vitis vinifera L.) using high resolution ancillary information. Precision Agriculture, 11(4), 358-378. https://doi.org/10.1007/s11119-010-9164-7

Acevedo-Opazo, C., Valdés-Gómez, H., Taylor, J. A., Avalo, A., Verdugo-Vásquez, N., Araya, M., ... Tisseyre, B. (2013). Assessment of an empirical spatial prediction model of vine water status for 
irrigation management in a grapevine field. Agricultural Water Management, 124, 58-68.

Arnó, J., Martínez-Casasnovas, J. A., Ribes-Dasi, M., \& Rosell, J. R. (2009). Review . Precision

Viticulture . Research topics, challenges and opportunities in site-specific vineyard management. Spanish Journal of Agricultural Research, 7(4), 779-790.

Arnó, J., Rosell, J. R., Blanco, R., Ramos, M. C., \& Martínez-Casasnovas, J. a. (2011). Spatial variability in grape yield and quality influenced by soil and crop nutrition characteristics. Precision Agriculture, 13(3), 393-410. https://doi.org/10.1007/s11119-011-9254-1

Baluja, J., Tardaguila, J., Ayestaran, B., \& Diago, M. P. (2013). Spatial variability of grape composition in a Tempranillo (Vitis vinifera L.) vineyard over a 3-year survey. Precision Agriculture, 14(1), 40-58. https://doi.org/10.1007/s11119-012-9282-5

Bonhomme, R. (2000). Bases and limits to using "degree.day" units. European Journal of Agronomy, 13(1), 1-10. https://doi.org/10.1016/S1161-0301(00)00058-7

Bramley, R. G. V., Evans, K. J., Dunne, K. J., \& Gobbett, D. L. (2011). Spatial variation in response to "reduced input" spray programs for powdery mildew and botrytis identified through whole-of-block experimentation. Australian Journal of Grape and Wine Research, 17(3), 341-350. https://doi.org/10.1111/j.1755-0238.2011.00153.x

Bramley, R. G. V., Ouzman, J., \& Boss, P. K. (2011). Variation in vine vigour, grape yield and vineyard soils and topography as indicators of variation in the chemical composition of grapes, wine and wine sensory attributes. Australian Journal of Grape and Wine Research, 17(2), 217-229. https://doi.org/10.1111/j.1755-0238.2011.00136.x

Caffarra, A., \& Eccel, E. (2010). Increasing the robustness of phenological models for Vitis vinifera cv. Chardonnay. International Journal of Biometeorology, 54(3), 255-67. https://doi.org/10.1007/s00484-009-0277-5

Chuine, I., García de Cortazar-Atauri, I., Kramer, K., \& Hänninen, H. (2013). Plant Development Models. In M. D. Schwartz (Ed.), Phenology: An Integrative Environmental Science (pp. 275-293). Dordrecht: Springer Netherlands. https://doi.org/10.1007/978-94-007-6925-0

Coombe, B. G. (1995). Adoption of a system for identifying grapevine growth stages. Australian Journal of Grape and Wine Research, 1(2), 104-110.

García de Cortázar-Atauri, I., Brisson, N., \& Gaudillere, J. P. (2009). Performance of several models for 
predicting budburst date of grapevine (Vitis vinifera L.). International Journal of Biometeorology, 53(4), 317-26. https://doi.org/10.1007/s00484-009-0217-4

Jorquera-Fontena, E., \& Orrego-Verdugo, R. (2010). Impact of Global Warming on the Phenology of a Variety of Grapevine Grown in Southern Chile. Agrociencia, 44(4), 427-435.

King, P. D., Smart, R. E., \& McClellan, D. J. (2014). Within-vineyard variability in vine vegetative growth , yield , and fruit and wine composition of Cabernet Sauvignon in Hawke 's Bay, New Zealand. Australian Journal of Grape and Wine Research, 20, 234-246. https://doi.org/10.1111/ajgw.12080

Kunz, T., \& Tatham, B. (2012). Localization in Wireless Sensor Networks and Anchor Placement. Journal of Sensor and Actuator Networks, 1(1), 36-58. https://doi.org/10.3390/jsan1010036

Matese, A., Crisci, A., Di Gennaro, S., Primicerio, J., Tomasi, D., Marcuzzo, P., \& Guidoni, S. (2014). Spatial variability of meteorological conditions at different scales in viticulture. Agricultural and Forest Meteorology, 189-190, 159-167. https://doi.org/10.1016/j.agrformet.2014.01.020

Molitor, D., Junk, J., Evers, D., Hoffmann, L., \& Beyer, M. (2013). A High-Resolution Cumulative Degree Day-Based Model to Simulate Phenological Development of Grapevine. American Journal of Enology and Viticulture, 1-33. https://doi.org/10.5344/ajev.2013.13066

Mullins, M. G., Bouquet, A., \& Williams, L. E. (1992). Biology of the Grapevine. New York, USA: Cambridge University Press. Retrieved from http://books.google.es/books?id=wnNvmRjfxgQC

Ortega-Farías, S., Lozano, P., Moreno, Y., \& León, L. (2002). Development of models for predicting phenology and evolution of madurity in cv. Cabernet Sauvignon and Chardonnay grapevines. Agricultura Técnica, 62(1), 27-37.

Parker, A., García de Cortázar-Atauri, I., Chuine, I., Barbeau, G., Bois, B., Boursiquot, J.-M., ... van Leeuwen, C. (2013). Classification of varieties for their timing of flowering and veraison using a modelling approach: A case study for the grapevine species Vitis vinifera L. Agricultural and Forest Meteorology, 180, 249-264. https://doi.org/10.1016/j.agrformet.2013.06.005

Parker, A. K., Garcia de Cortázar-Atauri, I., Van Leeuwen, C., \& Chuine, I. (2011). General phenological model to characterise the timing of flowering and veraison of Vitis vinifera L. Australian Journal of Grape and Wine Research, 17(2), 206-216. https://doi.org/10.1111/j.1755-0238.2011.00140.x

Primicerio, J., Matese, A., Gennaro, S. F. Di, Albanese, L., Guidoni, S., \& Gay, P. (2013). Development 
of an integrated, low-cost and open-source system for precision viticulture : from UAV to WSN. In EFITA-WCCA-CIGR Conference "Sustainable Agriculture through ICT Innovation" Turin, Italy (pp. 24-27).

Taylor, J. A., Coulouma, G., Lagacherie, P., \& Tisseyre, B. (2009). Mapping soil units within a vineyard using statistics associated with high-resolution apparent soil electrical conductivity data and factorial discriminant analysis. Geoderma, 153(1-2), 278-284. https://doi.org/10.1016/j.geoderma.2009.08.014

Taylor, J. A., Tisseyre, B., Bramley, R. G. V., \& Reid, A. (2005). A comparison of the spatial variability of vineyard yield in European and Australian production systems. In 5th European Conference on Precision Agriculture (pp. 907-915).

Tisseyre, B., Mazzoni, C., \& Fonta, H. (2008). Whithin-field temporal stability of some parameters in viticulture: Potential Toward a Site Specific Management. Journal International Des Sciences de La Vigne et Du Vin, 42(1), 27-39.

Verdugo-Vásquez, N., Acevedo-Opazo, C., Valdés-Gómez, H., Araya-Alman, M., Ingram, B., García de Cortázar-Atauri, I., \& Tisseyre, B. (2016). Spatial variability of phenology in two irrigated grapevine cultivar growing under semi-arid conditions. Precision Agriculture, 17(2), 218-245. https://doi.org/10.1007/s11119-015-9418-5

Verdugo-Vásquez, N., Pañitrur-De la Fuente, C., \& Ortega-Farías, S. (2017). Model development to predict phenological scales of table grapes (cvs. Thompson, Crimson and Superior Seedless and Red Globe) using growing degree days. Oeno One, 51(3), 277-288. https://doi.org/10.20870/oenoone.2017.51.2.1833 